\title{
Sudden Sensorineural Hearing Loss in a Hyperbaric Oxygen Chamber Employee Following Parotidectomy
}

\author{
Henry Ideker ${ }^{1}$, Ronald Walker ${ }^{1}$, and Joshua Sappington ${ }^{1}$ \\ ${ }^{1}$ Saint Louis University School of Medicine
}

November 15, 2021

\begin{abstract}
Sudden sensorineural hearing loss (SSNHL) is uncommon and oftentimes idiopathic. We describe the unique presentation of SSNHL in an HBO chamber employee following a parotidectomy, whose chief complaint was otalgia and aural fullness during dives. She ultimately recovered completely due to timely diagnosis and a high-dose steroid taper.

Sudden Sensorineural Hearing Loss in a Hyperbaric Oxygen Chamber Employee Following Parotidectomy

Running Title: SSNHL in HBO Employee Following Parotidectomy

Hank Ideker, $\mathrm{MD}^{1}$

Ronald J. Walker, $\mathrm{MD}^{1}$

Joshua M. Sappington, $\mathrm{MD}^{1}$

Corresponding Author:

Joshua M. Sappington, MD

1008 S. Spring $3^{\text {rd }}$ Floor

Saint Louis, MO 63104

Telephone: 314-977-4210

Fax: 314-977-1820

Email: joshua.sappington@health.slu.edu

${ }^{1}$ Department of Otolaryngology-Head and Neck Surgery Saint Louis University

No authors have any conflict of interest or financial disclosures for this study.

Written informed consent has been obtained by this patient, who has been de-identified, for publication.

IRB Statement: SLU IRB has determined that a descriptive report of observations on up to 5 people or organization(s), in which the observations were retrieved in a retrospective manner and no research questions/hypotheses are being tested, does not meet the definition of research, and may be considered a "case report". In such cases, SLU investigators are not required to obtain IRB approval prior to beginning the activity. However, investigators must make a reasonable attempt to protect the patient's/individual's privacy by removing identifiers.
\end{abstract}

Sudden Sensorineural Hearing Loss in a Hyperbaric Oxygen Chamber Employee Following Parotidectomy 
Key Words: Sudden Sensorineural Hearing Loss, SSNHL, Hyperbaric Oxygen Chamber, HBO, parotidectomy, otology

\section{Introduction:}

Sudden sensorineural hearing loss (SSNHL) is overall an unusual medical condition in the general population. However, within the field of otolaryngology it is commonly seen in all areas of practice and is an otologic emergency. Approximately $10 \%$ of such cases have a cause which is identifiable, and retrocochlear pathology must be ruled out with MRI; however, approximately $90 \%$ are idiopathic in nature. ${ }^{1}$

Idiopathic sudden sensorineural hearing loss (ISSNHL) is defined as acute hearing loss within 72 hours with a decrease of at minimum $30 \mathrm{~dB}$ in 3 consecutive frequencies on formal audiologic testing without an identifiable cause. ${ }^{1}$ This pathology most frequently presents unilaterally with associated tinnitus and aural fullness in the $5^{\text {th }}$ decade of life. Between 5 and 27 individuals per 100,000 per year are affected, which equates to approximately 66,000 new cases per year in the United States. ${ }^{1}$ This is most likely greatly underreported secondary to a percentage of patients who never seek care or recover hearing spontaneously.

There have been many studies analyzing treatments for ISSNHL, which are primarily high dose oral steroid taper and intratympanic steroid injection. ${ }^{2}$ Additionally hyperbaric oxygen has been investigated as an alternative therapy for ISSNHL either as primary or salvage treatment. ${ }^{3}$

\section{Case Report:}

We present an interesting case presentation of a 33 year-old female patient who underwent left parotidectomy for pleomorphic adenoma. She had a complete resection of the lesion with marvelous post-operative course without facial nerve issue or other complication. Interestingly she is employed at a regional hospital as a technician in the hyperbaric oxygen chamber. She returned to work initially without any issues. Fifteen days after surgery she noted slight left aural fullness and the inability to adequately pressurize her middle ear while at work, noting severe otalgia beyond 1 ATM.

These symptoms and acute change led her to present for an unscheduled clinic visit. On evaluation she was found to have a well-healing incision with intact facial nerve function. Micro-otoscopic exam was significant for an intact tympanic membrane with slight tympanosclerosis but without erythema, hemotympanum, effusion, tympanic membrane retraction, or vessel hemorrhage within the tympanic membrane. Unfortunately, as she lived approximately 90 miles from our tertiary academic medical center, she was seen late in the afternoon on a Friday. Formal audiologic services were not available, but examination with $512 \mathrm{~Hz}$ tuning fork was consistent with a left sided ASNHL with Weber lateralizing to the right and air conduction greater than bone conduction bilaterally. She was treated with high dose oral prednisone taper, and an MRI IAC with and without contrast was obtained to evaluate for retrocochlear pathology, which was ultimately negative.

Within days of starting the oral steroid taper she noted complete resolution of her left sided aural fullness and otalgia with hyperbaric oxygen dives. Additionally, she had formal audiologic testing following oral steroid therapy which demonstrated hearing within normal limits bilaterally with symmetric excellent word recognition scores.

\section{Discussion:}

This case is an interesting and unusual presentation of SSNHL for multiple reasons. Firstly, this pathophysiology had a close temporal relationship to an otolaryngologic procedure. While there are growing numbers of case reports involving SSNHL after general anesthesia in non-otolaryngologic procedures, there are limited reports involving otolaryngologic procedures. ${ }^{4}$ Secondly, her presentation due to her employment as a hyperbaric oxygen technician was unique. Her main presenting symptoms was otalgia and the inability to tolerate pressurization beyond 1 ATM. It is reasonable to believe that her parotidectomy was not the primary inciting event as it did not occur within the early post-operative period and she initially returned to work without any issue. It is however reasonable that her recent parotidectomy did play a small part in her presentation. Prior to her procedure she typically would use a phone on her left ear; however, as she was 
in the early postoperative period following parotidectomy she noted altered sensation of that ear and did not utilize a phone on that side. This likely contributed to a young healthy woman not noting a left-sided asymmetric hearing loss and rather noticing the aural fullness and inability to work beyond 1 ATM as her primary symptoms.

The most interesting aspect of her presentation is the inability to tolerate pressurization beyond 1 ATM during hyperbaric dives. This change was not due to eustachian tube dysfunction or the inability to perform a Valsalva as she demonstrated a well aerated middle ear and had a normal microscopic examination. The most common otologic complication with hyperbaric oxygen therapy is middle ear barotrauma. ${ }^{5}$ On examination this young woman did not demonstrate any findings consistent with middle ear barotrauma. This begs the question as to why was she unable to tolerate compression beyond 1 ATM? A possible explanation may be related to the multitude of nerves which provide innervation to the pinna, external auditory canal, tympanic membrane, and middle ear chemoreceptors which are all present in highly confined anatomy. It is extremely likely that this young lady had altered sensation on the left side owing to both the recent parotidectomy and possible neural dysfunction stemming from the ISSNHL. These sensory changes and neural dysfunction may have led to a significant alteration in perception leading to otalgia with pressurization despite normal middle ear and eustachian tube function. Ultimately, the exact pathophysiology may never be elucidated but a high index of suspicion should be in place for suspected cases of ISSNHL.

\section{Conclusion:}

This case is a unique presentation of ISSNHL, owing to recent surgical history of parotidectomy and occupation in a hyperbaric oxygen chamber. Given her late consultation on Friday afternoon, formal audiologic testing was not available and ISSNHL was confirmed via tuning forks. With this confirmation, high dose oral steroids were prescribed and a contrasted MRI was obtained to rule out retrocochlear pathology. She did well with appropriate treatment and had complete resolution of her symptoms, improvement in hearing to baseline and ability to perform her job as a hyperbaric oxygen technician. Regardless of the presentation, ISSNHL is a true otologic emergency requiring high index of suspicion, early recognition, and low threshold to treat.

\section{References}

1. Chandrasekhar SS, Tsai Do BS, Schwartz SR, et al. Clinical Practice Guideline: Sudden Hearing Loss (Update). Otolaryngol Head Neck Surg . 08 2019;161(1_suppl):S1-S45. doi:10.1177/0194599819859885

2. Mirian C, Ovesen T. Intratympanic vs Systemic Corticosteroids in First-line Treatment of Idiopathic Sudden Sensorineural Hearing Loss: A Systematic Review and Meta-analysis. JAMA Otolaryngol Head Neck Surg . May 2020;146(5):421-428. doi:10.1001/jamaoto.2020.0047

3. Rhee TM, Hwang D, Lee JS, Park J, Lee JM. Addition of Hyperbaric Oxygen Therapy vs Medical Therapy Alone for Idiopathic Sudden Sensorineural Hearing Loss: A Systematic Review and Meta-analysis.JAMA Otolaryngol Head Neck Surg . 12 2018;144(12):1153-1161. doi:10.1001/jamaoto.2018.2133

4. Punj J, Pandey R, Darlong V. Sensorineural hearing loss after general anaesthesia: 52 cases reported until now! Anaesthesia . Feb 2009;64(2):226. doi:10.1111/j.1365-2044.2008.05846.x

5. Lima MA, Farage L, Cury MC, Bahamad F. Update on middle ear barotrauma after hyperbaric oxygen therapy-insights on pathophysiology.Int Arch Otorhinolaryngol . Apr 2014;18(2):204-9. doi:10.1055/s-00341366974 